\title{
Universality Class of Thermally Diluted Ising Systems at Criticality
}

\author{
Manuel I. Marqués and Julio A. Gonzalo \\ Depto. de Física de Materiales C-IV \\ Universidad Atónoma de Madrid \\ Cantoblanco 28049 Madrid Spain \\ email: julio.gonzalo@uam.es \\ Jorge Íñiguez \\ Depto. de Física Aplicada II \\ Universidad del Pais Vasco \\ Apdo 64448080 Bilbao Spain
}

(25-1-2000)

\begin{abstract}
The universality class of thermally diluted Ising systems, in which the realization of the disposition of magnetic atoms and vacancies is taken from the local distribution of spins in the pure original Ising model at criticality, is investigated by finite size scaling techniques using the Monte Carlo method. We find that the critical temperature, the critical exponents and therefore the universality class of these thermally diluted Ising systems depart markedly from the ones of short range correlated disordered systems. Our results agree fairly well with theoretical predictions previously made by Weinrib and Halperin for systems with long range correlated disorder.
\end{abstract}

\section{Introduction}

During last decades the systems with quenched randomness have been intensively studied [1]. The Harris criterion [2] predicts that weak dilution does not change the character of the critical behavior near second order phase transitions for systems of dimension $d$ with specific heat exponent lower than zero in the pure case (the so called $\mathrm{P}$ systems), $\alpha_{\text {pure }}<0 \Longrightarrow \nu_{\text {pure }}>2 / d$, being $\nu$ the correlation length critical exponent. This criterion has been supported by renormalization group (RG) [3, 4. 5], and scaling analyses [6]. The effect of strong dilution was studied by Chayes et al. [7]. For $\alpha_{\text {pure }}>0$ (the so called $\mathrm{R}$ systems), the Ising $3 \mathrm{D}$ case for example, the system fixed point flows from a pure (undiluted) fixed point towards a new stable fixed point [3, [, 5, 6, 6, 8] at which $\alpha_{\text {random }}<0$. Recently Ballesteros et al. have used the Monte Carlo approach to study the diluted Ising systems in two [9], three [10] and four dimensions [11]. The existence of a new universality class for the random diluted Ising system (RDIS), different from that of the pure Ising model and independent of the average density of occupied spin states $(p)$, is proved, using an infinite volume extrapolation technique [10] based upon the leading correction to scaling. The critical exponents obtained this way could be compared with the experimental critical exponents for a random disposition of vacancies in diluted magnetic systems [12].

In all cases previously mentioned frozen disorder was always produced in a random way, that is, vacancies were distributed throughout the lattice randomly. Real systems, however, can be realized with other kinds of disorder, where the vacancy locations are correlated. In particular, long range correlation (LRC) has been found in X-ray and Neutron Critical Scattering experiments in systems undergoing magnetic and structural phase transitions 13, 14]. This effect has been modeled by assuming a spatial distribution of critical temperatures obeying a power law $g(x) \sim x^{-a}$ for large separations $x$ 15. In general these systems behave in a way very different from RDIS, since systems with randomly distributed impurities may be considered as the limit case of short range correlated (SRC) distributions of the vacancies. The basic approach to the critical phenomena of LRC systems was established by Weinrib and Halperin [16] almost two decades ago. They found that the Harris criterion can be extended for these cases, showing that for $a<d$, the disorder is irrelevant if $a \nu_{\text {pure }}-2>0$, and that in the case of relevant disorder a new universality class (and a new fixed point) with correlation length exponent $\nu=2 / a$, and a specific heat exponent $\alpha=2(a-d) / a$ appears. In contrast, if $a>d$, the usual Harris criterion for SRC systems is recovered. LRC disorder has been studied also by the Monte Carlo approach [17]. In this case a correlation function $g(x)=x^{-a}$ with $a=2$ (defects consisting in randomly oriented lines of magnetic vacancies inside a three dimensional Ising system) confirmed the theoretical predictions of Weinrib and Halperin.

In the present paper we will study Ising three dimensional systems where the long range correlated dilution has been introduced as a thermal order-disorder distribution of vacancies in equilibrium, governed by a characteristic ordering temperature $(\theta)$, in a way similar to the thermal disordering in a binary alloy of magnetic (spins) and non-magnetic atoms (vacancies). [See previous work [18] and also the application of this kind of 
disorder in percolation problems [19]]. Making more explicit the analogy with order-disorder in alloys we may distinguish clearly between:

i) thermally diluted Ising system realizations (TDIS), in which the quenched randomness is produced by considering a ferromagnetic Ising system at $(\theta=$ $\left.T_{c}^{3 D}\right)$ : after thermalization, the spins of the dominant type (concentration $c \geq 0.5$ ) are taken as the locations of the magnetic atoms, and the rest are taken as the magnetic vacancies. The structure of the realization is fixed thereafter for all temperatures at which the magnetic interactions are subsequently investigated.

ii) random diluted Ising system realizations (RDIS) (equivalent to $\theta>>T_{c}^{3 D}$ ), also fixed thereafter for all temperatures at which the spin interactions are investigated. Of course, we chose for comparison a vacancy probability $p=0.5$, resulting in $c \approx 0.5$.

iii) as a third possibility, not considered here, one could investigate what might be called an antiferromagnetically diluted Ising system (with $\theta<<T_{N}^{3 D}$, which would lead to a disposition of non-magnetic atoms (vacancies) strictly alternating with spins, with $c=0.5$.

So, if this ordering temperature $\theta$ determining the particular realization is high enough, the equilibrium thermal disorder will be very similar to the random (short range correlated) disorder of the usual previous investigations. On the other hand, if $\theta$ happens to coincide with the characteristic magnetic critical temperature $\left(T_{c}^{3 D}=4.511617\right)$ of the undiluted system, we will have vacancies in randomly located points, but with a long range correlated distribution. [Note that the situation differs markedly from that of previously studied LRC systems, in which lines or planes of vacancies were considered]. The correlation of our TDIS is given by a value $a=2-\eta_{\text {pure }}$, where $\eta_{\text {pure }}$ is the correlation function exponent for the pure system. Since $d=3$ and $\eta_{\text {pure }}=0.03$ for the three dimensional Ising system, we have a long range correlated disorder with $a=1.97<3=d$. So we are in the case where LRC disorder is relevant and we should detect a change of universality class with respect to the SRC case (following Weinrib and Halperin we expect for the thermally diluted Ising system $\nu \approx 1$ and $\alpha \approx-1)$. Details about the construction of these thermally diluted Ising systems (TDIS) can be found in Ref. [18]. In the present work we study the critical behavior and the university class of three dimensional TDIS at criticality using the Monte Carlo approach. We will compare our results with the critical behavior of the RDIS.

The structure of the paper is as follows: In Section II we study the dependence of the critical temperature (and of the self-averaging at criticality) with the size of the system for both TDIS and RDIS. Once the critical point is determined, we investigate whether or not TDIS and RDIS belong to the same universality class. In order to proceed, we study the critical behavior of both kinds of systems by applying finite size scaling techniques (Section
III) and by using the effective-exponent approach (Section IV). A summary of the main results and concluding remarks are given in Section $\mathrm{V}$.

\section{Transition temperature and self-averaging of thermally diluted systems}

For a hypercubic sample of linear dimension $L$ and number of sites $N=L^{d}$, any observable singular property $X$ has different values for the different random realizations of the disorder, corresponding to the same dilution probability $p$ (grand-canonical constraint). This means that $\mathrm{X}$ behaves as a stochastic variable with average $\bar{X}$ (in the following, the bar indicates average over subsequent realizations of the dilution and the brackets indicate MC average). The variance would then be $(\Delta X)^{2}$, and the normalized square width, correspondingly:

$$
R_{X}=(\Delta X)^{2} / \bar{X}^{2}
$$

A system is said to exhibit self-averaging (SA) if $R_{X} \rightarrow 0$ as $L \rightarrow \infty$. If the system is away from criticality, $L>>\xi$ (being $\xi$ the correlation length). The central limit theorem indicates that strong SA must be expected in this case. However, the behavior of a ferromagnet at criticality (with $\xi>>L$ ) is not so obvious. This point has been studied recently for short range correlated quenched disorder. Aharony and Harris (AH), using a renormalization group analysis in $d=4-\varepsilon$ dimensions, proved the expectation of a rigorous absence of self-averaging in critically random ferromagnets [20]. More recently, Monte Carlo simulations were used to investigate the self-averaging in critically disordered magnetic systems [10,21,22]. The absence of self-averaging was confirmed. The normalized square width $R_{X}$ is an universal quantity affected just by correction to scaling terms. LRC diluted systems are expected to have different critical exponents and different normalized square widths with respect to those of the usual randomly disordered systems studied previously.

We perform Monte Carlo calculations of the magnetization and the susceptibility $\left(\chi=\left(\left\langle M^{2}\right\rangle-\langle M\rangle^{2}\right) / T\right)$ per spin at different temperatures for different realizations of TDIS, and for randomly diluted systems with $p=0.5$ (restricting to $c>0.5$ ) using in both cases the Wolff 23 single cluster algorithm 24 with periodic boundary conditions, on lattices of different sizes $L=10,20,40,60,80,100$. Results for susceptibility vs. temperature are shown in Fig. 1. 

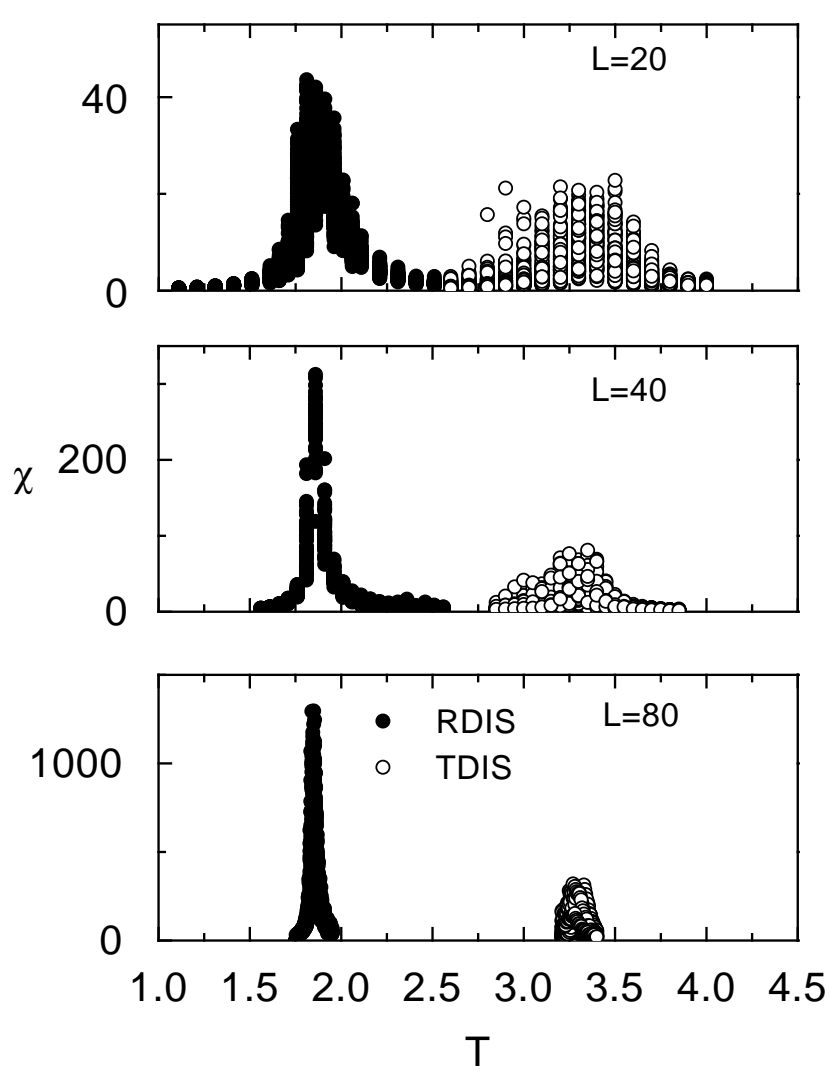

FIG. 1. Susceptibility $\chi$ vs. temperature $T$ for random dilution realizations $(p=0.5)$ (black) and critical thermal dilution realizations (white). The size of the systems under consideration is given by $L=20,40,80$.

Note how due to the existence of randomness the susceptibility points do not collapse into a single curve, since each realization has a different value of the critical temperature and of the concentration. This is even more clear for small values of $\mathrm{L}$ and for the critically thermal case (white points). Fig. 1 indicates that the critical temperature of the TDIS clearly differs from that of the RDIS, and also than the effect of the dilution on the lack of self-averaging seems to be stronger in the thermally diluted Ising case. The critical temperature may be obtained at the point where the normalized square width for the susceptibility, $R_{\chi}$, reaches its maximum. A different value of the critical temperature does not imply, of course, a different universality class. However from the dependence of the critical temperature with the length of the system we expect to detect a change in universality between TDIS and RDIS following the scaling relation:

$$
T_{c}(L)=T_{c}(\infty)+A L^{-1 / \nu}
$$

being $\nu$ the critical exponent associated with the specific system's correlation length. This critical exponent has been determined by means of Monte Carlo data for the random case by Ballesteros et al. [10]. They found a value $\nu_{\text {random }}=0.683$. On the other hand the result by Weinrib and Halperin [16] indicates that the critical exponent expected for the thermal case should be $\nu_{\text {thermal }}=2 / a=1.015$. Fig. 2 represents the dependence of the critical temperature with respect to the length of the systems for random and thermal dilutions.
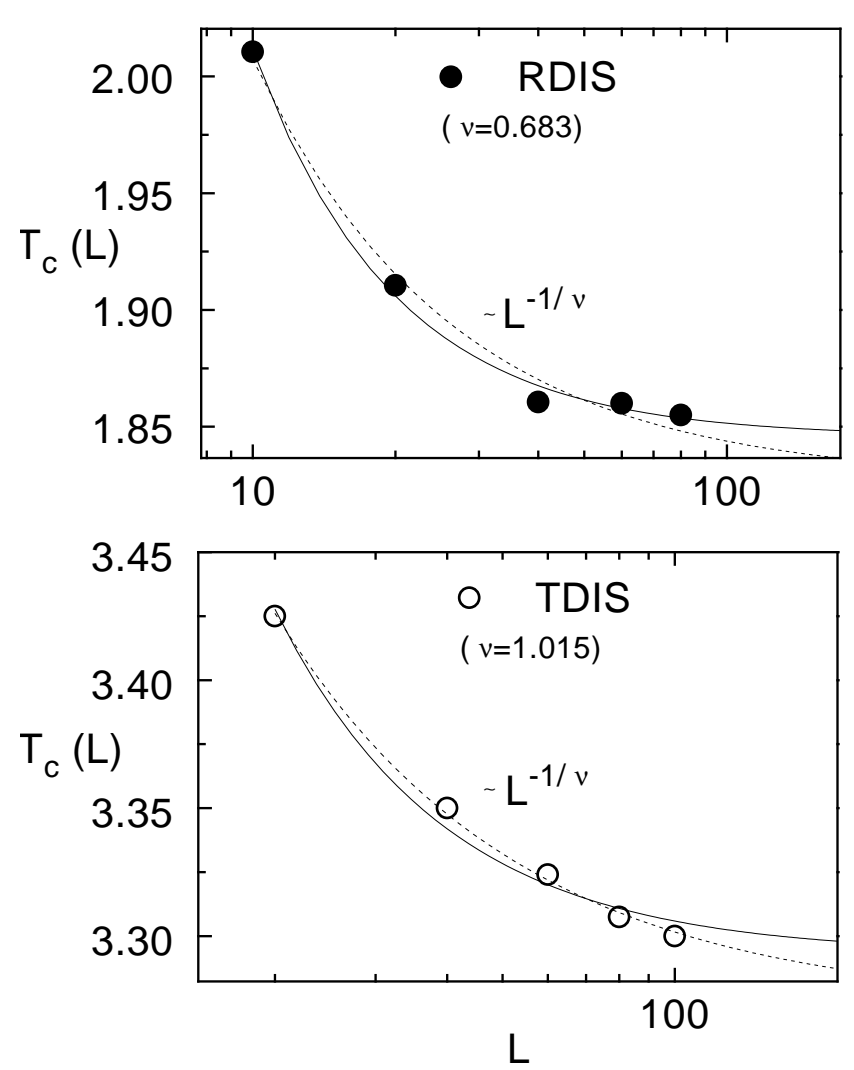

FIG. 2. Semi log representation of critical temperature $T_{c}$ vs. lenght $L$ for random dilution $(p=0.5)$ (black) and for thermal dilution (white). The continuous line is the fit obtained using $\nu=0.6837$ (short range correlated random exponent) and dashed line the fit obtained using $\nu=1.015$ (long range correlated exponent, $\mathrm{a}=1.97)$ in either case.

In both cases a fit to Eq. 2 has been performed for both values $\nu=\nu_{\text {random }}=0.683$ (continuous line) and $\nu=\nu_{\text {thermal }}=1.015$ (dashed line). Note how the thermal data fit better Eq. 2 for $T_{c}(L)$ using $\nu_{\text {thermal }}$, indicating a possible change in universality class with respect to the random case. [If we fit the data leaving all parameters free, we find $\nu_{\text {random }} \approx .0 .7$ and $\nu_{\text {thermal }} \approx 1.2$, which are very near the expected results]. The extrapolated values of critical temperatures for infinite systems obtained this way are $T_{c}^{\text {random }}(\infty)=1.845 \pm 0.003$ (close to the values previously obtained by Ballesteros et al. 
[10]) and $T_{c}^{\text {thermal }}(\infty)=3.269 \pm 0.002$ (clearly different from $T_{c}(\infty)$ for the SRC case). Incidentally $\nu_{\text {thermal }}$ can be compared with $\nu$ for the observed sharp component in neutron scattering line shapes, which is around 1.3 for $\mathrm{Tb}$ [14]. This point deserves more careful analysis and will be taken up elsewhere.

Once the critical temperatures are known we can perform simulations for the magnetization and the susceptibility at criticality for several realizations of thermal and random diluted systems in an effort to determine the value of $R_{\chi}$, the normalized square width for the susceptibility. We went up to 500 realizations for $L=10,20,40$ and up to 200 realizations for $L=60,80$. Results are shown in Fig.3.

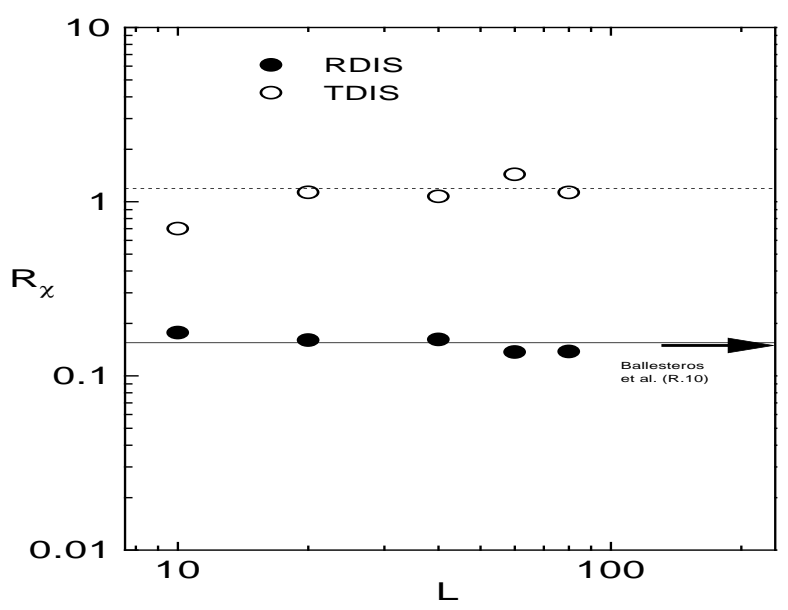

FIG. 3. Log-log plot of the normalized square width for the susceptibility at criticallity $R_{\chi}$ vs. lenght $L$ for random dilution $(p=0.5)$ (black) and thermal dilution (white).

The arrow represents the (concentration independent) $R_{\chi}$ value obtained by Ballesteros et al [10]. The straight continuous line represents the average value obtained for random dilution data, and the straight dashed line gives the average value obtained for thermal dilution data. Note that the TDIS presents a lack of self-averaging around one order of magnitude larger than the RDIS. We have already presented a similar analysis for both kinds of dilution 18], but only at the critical temperature characteristic of the random system. Our results are not precise enough to specify accurately the evolution of the normalized square width as a function of $L$ governed by corrections to scaling terms. However the average we obtain for $R_{<}^{\text {random }}=0.155$ is close to the value previously reported [10] by means of infinite volume extrapolations. For the thermal case we obtain $R_{\chi}^{\text {thermal }}=1.19$, about one order of magnitude larger than for random dilution. In this case an evolution of $R_{\chi}^{\text {thermal }}$ vs. $L$ given by correction to scaling terms may be also expected, but ac- cording to Weinrib and Halperin [16] the analysis would be even more complicated, due to the fact that the long range correlated disordered systems present complex oscillating corrections to scaling.

\section{Critical behavior and exponents of ther- mally diluted systems}

The dispersion in concentration and magnetization at criticality between the different realizations is shown at a glance in scattered plots as in Ref. 18. Each point in Fig. 4 represents a single realization with magnetization at criticality $(M)$ and concentration $(c)$. Note that in both cases (TDIS and RDIS), the dispersion on the magnetization and on the concentration decreases with $\mathrm{L}$, but this is more clearly shown in the thermal case. Fig. 4 shows clearly the difference in behavior between the random and the thermal cases, at least up to the values of $L$ considered.

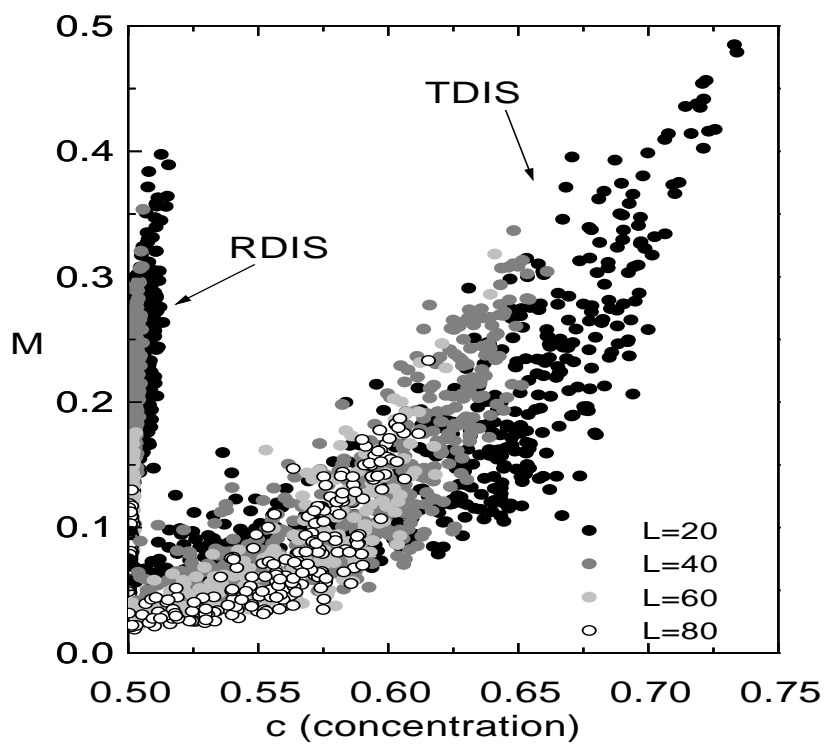

FIG. 4. Scattered plot of magnetization $M$ at the critical temperature vs. concentration $c$ for the realizations considered of the random $(p=0.5)$ and the thermal dilutions. $(L=20,40,60,80)$.

From Fig. 4 we can extract averaged values for the magnetization and the inverse susceptibility, $\bar{M}$ and $\overline{\chi^{-1}}$ (with $\chi=\left\langle M^{2}\right\rangle$ at the critical point) for the TDIS. Both averaged values are expected to fit the following scaling laws at criticality:

$$
\begin{gathered}
\overline{M(L)} \propto L^{-\beta / \nu} \\
\overline{\chi^{-1}(L)} \propto L^{-\gamma / \nu}
\end{gathered}
$$

Considering $(1 / \nu)_{\text {thermal }}=a / 2=0.985$, we can ob- 
tain from our data the values of $\beta_{\text {thermal }}$ and $\gamma_{\text {thermal }}$ (see f.i. the fitting for the inverse of the susceptibility in Fig. 5). We get $\beta_{\text {thermal }}=0.56 \pm 0.05$ and $\gamma_{\text {thermal }}=1.91 \pm 0.06$, very close to the predicted values by Weinrib and Halperin [16]. Using the scaling relation:

$$
\alpha=2-2 \beta-\gamma
$$

we obtain the following specific heat critical exponent: $\alpha_{\text {thermal }}=-1 \pm 0.1$. Weinrib and Halperin give for LRC systems [16] $\alpha=-1$, in good agreement with our result. An analogous analysis has been performed but using the dispersion in magnetization and inverse susceptibility instead of the averaged values. The results are similar. The same study has been made for the random case (also shown in Fig.5).

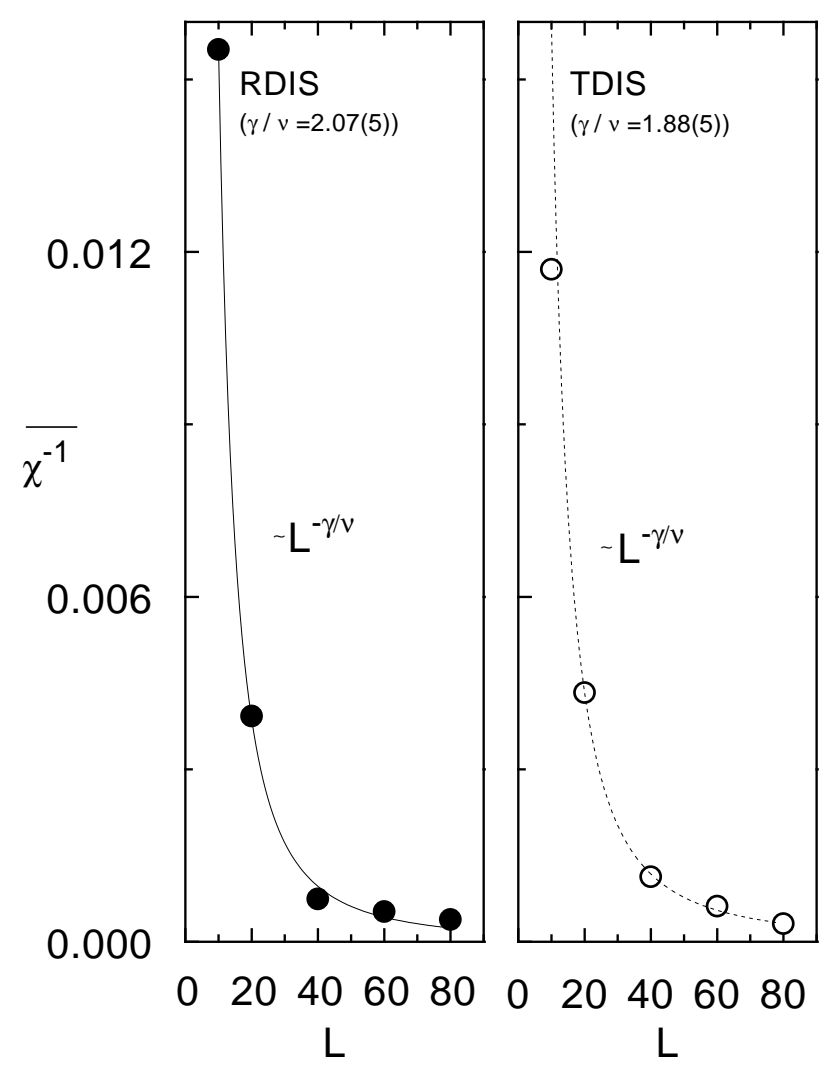

FIG. 5. Average value of the inverse of the susceptibility $\overline{\chi^{-1}(L)}$ vs. the lenght of the system $L$ for the random dilution $(p=0.5)$ case (black) and the thermal dilution case (white). Continuous line and dashed line indicate fits to the random and the thermal cases, respectively.

The critical exponents obtained are in agreement with those of Ballesteros et al. [10] within our error bars.

The average concentration for the thermal system is also expected to show a scaling law behavior given by:

$$
\overline{(c(L)}-0.5) \propto L^{-(\beta / \nu)_{3 D}}
$$

where $(\beta / \nu)_{3 D} \simeq 0.52$ gives the values corresponding to the pure three dimensional Ising case, because in critically thermally diluted Ising systems, vacancies are distributed with the same long range correlation spin distribution function as in the pure case [18]. The fit to the average value of the concentrations shown in Fig. 4 give, for the thermal case, a value $(\beta / \nu)_{3 D} \simeq 0.55 \pm 0.08$. This implies also a clear difference between RDIS and TDIS, since Ising systems with vacancies randomly distributed are not expected to follow an scaling behavior with $(\beta / \nu)_{3 D}$. [Fitting to an scaling law the results for RDIS gives an exponent around 1.4, which implies a much faster convergence to $c=0.5]$.

\section{Effective exponents and possible crossover for finite systems}

The difference between the universality class of RDIS and TDIS can be detected also by means of the effective critical exponents. In the case of the magnetization the effective critical exponent is defined by:

$$
\beta_{\text {eff }} \equiv \partial \log (M) / \partial \log (t) \quad\left(t=T_{c}^{i}-T\right)
$$

with $T_{c}^{i}$ the critical temperature of the particular realization (i) characterized by a maximum of the susceptibility $\left(\chi=\left\langle M^{2}\right\rangle-\langle M\rangle^{2}\right)$. For $L \rightarrow \infty$ and $t \rightarrow 0$, $\beta_{\text {eff }}=\beta$. Finite size effects force the effective critical exponent to drop to zero before the critical value is attained. However, since $\beta_{\text {random }}=0.3546$ (Ref. [10]), and $\beta_{\text {thermal }}$ is expected to be around 0.5 (Ref. [16]) , effective critical exponents (for the thermal case) may rise to values greater than 0.3546 and lower than 0.5 , before finite size effects appear, indicating the difference of universality class between both kinds of systems. In the RDIS the effective critical exponent is expected to be always lower than 0.3546. Monte Carlo simulations of magnetization vs. temperature have been performed for randomly $(p=0.5)$ and thermally diluted systems, with $L=80$. In Fig. 6 we show the results for $\beta_{\text {eff }}$ vs. $\log (t)$ for two samples of the TDIS and the RDIS type respectively. The random effective critical exponent is always under 0.35 and it seems to tend towards this value for large enough $L$, as expected, but for thermal systems the behavior is completely different: In the figure the value of the critical thermal effective exponent is between 0.35 and 0.5. An analogous investigation has been done for different values of $\mathrm{L}$ and different realizations. The same effect has been found for lower $\mathrm{L}$ values. However, we may note that in TDIS the effective critical exponent arrives at the maximum in a very different way depending on the realization. The reason is twofold: (1) the different disposition of the vacancies in each particular realization, and (2) the large differences 
in concentration for the thermal case (the rise of $\beta_{\text {eff }}$ towards the expected diluted universality value should be faster for c closer to 0.5). It may be noted that as the size $(L)$ of the sample increases the possibility of local inhomogeneities in the TDIS realizations increases, giving rise to such phenomena as pseudo double peaks in the susceptibility, reduced values for the overall critical exponents, etc. However, it is important to remark than in all realizations investigated the $\beta_{\text {eff }}$ values of the TDIS, (before finite size effects take over), have been clearly superior to the $\beta$ for the RDIS.

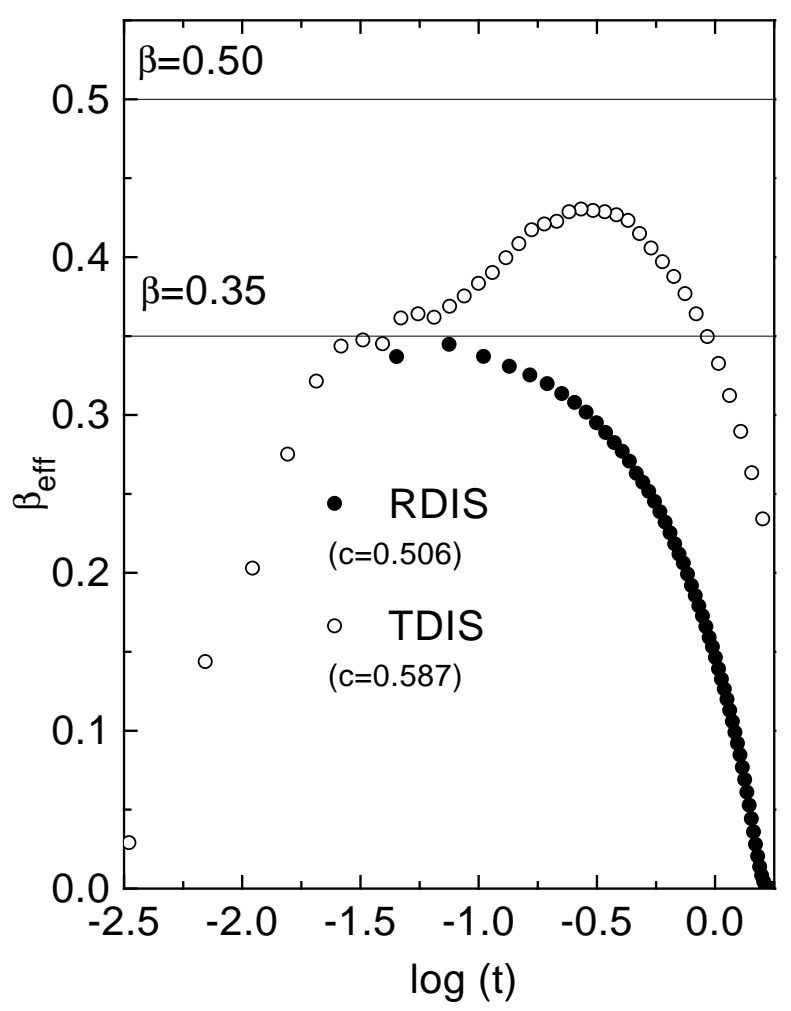

FIG. 6. Effective critical magnetization exponent $\beta_{\text {eff }}$ vs. $\log (t)$, with $t=T_{c}^{i}-T$, for the thermal dilution case (white) and the random dilution $(p=0.5)$ case (black). The realizations considered correspond to $L=80$.

In Fig. 6 the effective critical exponent for the thermal case seems to produce a crossover towards $\beta=0.35$ as the temperature approaches the critical point, before finite size effects finally appear. In principle this might be influenced by the indetermination in the measurement of the particular critical temperature of the realization. However, this crossover may point out that different length scales might be important at different distance from the critical point. In principle characteristic lengths such as the size of the fixed vacancies and spin clusters, the size of the system itself and the thermal spin fluctuations might all play a role. The possibility that for $L \longrightarrow \infty$ and $c=0.5$ the apparent crossover could disappear all together should not be excluded, entailing no crossover from critical thermal to random universality class.

\section{Concluding remarks}

To summarize, a new way to produce diluted Ising systems with a long range correlated distribution of vacancies has been analyzed by means of Monte Carlo calculations and finite size scaling techniques. We find an universality class different from that reported for diluted Ising systems with short range correlated disorder. Our systems may be included in the universality class predicted by Weinrib and Halperin for $a \approx 2$. This kind of thermal disorder had been already applied in percolation problems, but as far as we know it had never been applied to magnetic systems, in which long range correlated disorder has been previously produced mostly by random distribution of lines or planes of vacancies. The present dilution procedure may be applicable to systems where the long range correlated disorder is not due to dislocations, preferential lines or planes of vacancies, but to systems where the vacancies (points) are critically distributed in clusters as in the case of order-disorder systems.

We thank H.E. Stanley for encourament and we are grateful to H.G. Ballesteros, L.A. Fernández, V. MartínMayor, and A. Muñoz Sudupe for helpful correspondence. We thank P.A. Serena for helpful comments and for generous access to his computing facilities. We acknowledge financial support from DGCyT through grant PB960037 and from the Basque Regional Government (J.I.).

[1] For a review, see R.B. Stinchcombe, in Phase Transitions and Critical Phenomena, edited by C. Domb and J.L. Lebowitz (Academic, New York, 1983), Vol. 7.

[2] A. B. Harris, J. Phys. C 7, 1671 (1974).

[3] T. C. Lubensky and A. B. Harris, in Magnetism and Magnetic Materials, edited by C. D. Graham, G. H. Lander and J. J. Rhyne, AIP Conf. Proc. No. 24 (AIP, New York, 1975), p.99; A. B. Harris and T. C. Lubensky, Phys. Rev. Lett. 33, 1540 (1974).

[4] T. C. Lubensky, Phys. Rev. B 11, 3573 (1975).

[5] G. Grinstein and A. Luther, Phys. Rev. B 13, 1329 (1976).

[6] A. Aharony, in Phase Transitions and Critical Phenomena, edited by C. Domb and M.S. Green (Academic, New York, 1976), Vol. 6, p. 357.

[7] J. T. Chayes, L. Chayes, D. S. Fisher, and T. Spencer, Phys. Rev. Lett. 57, 2999 (1986). 
[8] W. Kinzel and E. Domany, Phys. Rev. B 23, 3421 (1981); D. Andelman and A. N. Berker, Phys. Rev. B 29, 2630 (1984).

[9] H. G. Ballesteros, L. A. Fernández, V. Martín-Mayor, A. Muñoz Sudupe, G. Parisi and J. J. Ruiz-Lorenzo, J. Phys. A 30, 8379 (1997).

[10] H. G. Ballesteros, L. A. Fernández, V. Martín-Mayor, A. Muñoz Sudupe, G. Parisi and J. J. Ruiz-Lorenzo, Phys. Rev. B 58, 2740 (1998).

[11] H. G. Ballesteros, L. A. Fernández, V. Martín-Mayor, A. Muñoz Sudupe, G. Parisi and J. J. Ruiz-Lorenzo, Nucl. Phys. B 512[FS], 681 (1998).

[12] R. Folk, Yu. Holovatch and T. Yavors'kii, condmat/9909121.

[13] T. R. Thurston, G. Helgesen, D. Gibbs, J. P. Hill, B. D. Gaulin and G. Shirane, Phys. Rev. Lett. 70, 3151 (1993); T. R. Thurson, G. Helgesen, J. P. Hill, D. Gibbs, B. D. Gaulin and P. J. Simpson, Phys. Rev. B 49, 15730 (1994).

[14] P. M. Gehring, K. Hirota, C. F. Majkrzak and G. Shirane, Phys. Rev. Lett. 71, 1087 (1993); K. Hirota, G. Shirane, P.
M. Gehring and C. F. Majkrzak, Phys. Rev. B 49, 11967 (1994).

[15] M. Altarelli, M.D. Núñez-Regueiro and M. Papoular, Phys. Rev. Lett. 74, 3840 (1995).

[16] A. Weinrib and B. I. Halperin, Phys. Rev. B 27, 413 (1983).

[17] H. G. Ballesteros and G. Parisi, cond-mat/9903230.

[18] M. I. Marqués and J. A. Gonzalo, Phys. Rev. E 60, 2394 (1999).

[19] W. Klein, H. E. Stanley, P.J. Reynolds and A. Coniglio, Phys. Rev. Lett. 41, 1145 (1978).

[20] A. Aharony and A. B. Harris, Phys. Rev. Lett. 77, 3700 (1996).

[21] S. Wiseman and E. Domany, Phys. Rev. Lett. 81, 22 (1998).

[22] A. Aharony, A. B. Harris and S. Wiseman, Phys. Rev. Lett 81, 252 (1998).

[23] U. Wolff, Phys. Rev. Lett. 62, 361 (1989).

[24] S. Wang and R. H. Swendsen Physica (Amsterdam) 167A, 565 (1990). 